Original paper

\title{
EFOMP survey results on national radiotherapy dosimetry audits
}

\author{
Oscar Casares-Magaz ${ }^{\text {a, }}$, Loredana G. Marcu ${ }^{\text {b,c }}$, Yolanda Prezado ${ }^{\text {d }}$, Efi Koutsouveli ${ }^{\text {e }}$, \\ Marco Brambilla ${ }^{\mathrm{f}}$ \\ ${ }^{a}$ Department of Medical Physics - Oncology, Aarhus University Hospital, Aarhus, Denmark \\ ${ }^{\mathrm{b}}$ Faculty of Informatics \& Science, University of Oradea, Oradea, Romania \\ ${ }^{\mathrm{c}}$ Cancer Research Institute, University of South Australia, Adelaide, Australia \\ ${ }^{\mathrm{d}}$ Institut Curie, Université PSL, CNRS UMR3347, Inserm U1021, 91400 Orsay, France \\ e Department of Medical Physics, Hygeia Hospital, Athens, Greece \\ ${ }^{\mathrm{f}}$ Health Physics Department, University Hospital, Novara, Italy
}

\section{A R T I C L E I N F O}

\section{Keywords:}

Dosimetry audits

Council directive

Radiotherapy quality assurance

Radiotherapy

National regulations

\begin{abstract}
A B S T R A C T
Purpose: The Council Directive 2013/58/EURATOM entered into force in 2014, and its transposition into national legislations became applicable in 2018. The Council Directive 2013/58/EURATOM strengthened the importance of clinical audits, and stated that Member States should ensure dosimetry audit compliance in accordance with national procedures. Therefore, the purpose of this work was to picture the status of the implementation of dosimetry audits in European countries. Methods: A questionnaire was designed to describe dosimetry audit standards in radiotherapy across European countries. The questionnaire was sent to $33 \mathrm{EFOMP}$ National Member Organizations (NMO). Results: Nineteen NMOs responded to the survey (14 EU members). For $58 \%$ of the participating countries national regulations required dosimetry audits in radiotherapy departments. In $37 \%$ of the participating countries there were implemented regulations for independent/secondary dose verification, and in $21 \%$ of the participating countries similar procedures for dose verification were already implemented although not regulated by law. In $42 \%$ of the participating countries there were implemented mechanisms to review updates and advances in the field of radiotherapy.

Conclusions: The transposition and further implementation of the Council Directive 2013/59/EURATOM was scarce, leading to heterogeneities in national policies about dosimetry audits.
\end{abstract}

\section{Introduction}

Efomp

The European Federation of Organisations for Medical Physics (EFOMP) was founded in May 1980 in London to serve as an umbrella organisation for all National Member Organisations (NMOs). The current membership covers 36 national organisations (26 EU members and 10 non-EU) which together represent more than 9000 medical physicists and clinical engineers working in the field of medical physics. The aims and purposes of the Federation include: foster exchanging of the educational, scientific, training and professional activities of NMOs in the field of Medical Physics; formulate common policies and guidelines; encourage safe application of ionising and non-ionising radiations or other physical agents in medicine; and forge links with other organisations where common interests are shared.
The 2013/59/EURATOM Council Directive [1] entered into force in 2014, and the transposition into national legislations came applicable in 2018. The Council Directive updated basic radioprotection standards in clinical and professional environments. In comparison with Council Directive 97/43/Euratom of 30 June 1997 [2], the new Council Directive 2013/59/EURATOM gave a stronger emphasis on clinical audits by establishing that "clinical audits are carried out in accordance with national procedures" (art 58(e)). The clinical audit was defined as "a systematic examination or review of medical radiological procedures which seeks to improve the quality and outcome of patient care through structured review, whereby medical radiological practices, procedures and results are examined against agreed standards for good medical radiological procedures, with modification of practices, where appropriate, and the application of new standards if necessary".

Dosimetry audits are one of several measures put in place to ensure patient safety in radiotherapy (RT). In international clinical trials, many

\footnotetext{
* Corresponding author at: Department of Medical Physics - Oncology, Aarhus University Hospital, Denmark.

E-mail address: oscar.casares@gmail.com (O. Casares-Magaz).
} 
clinical trials organisations require a recent dosimetry audit for trial participation [3], therefore accurate dosimetry quality assurance (QA) and quality control (QC) doubles as a tool against subtle machinerelated bias.

Performing an external audit is expensive and requires dedicated time and effort; in some countries the basic safety standards mandate regular audits, with varying requirements and frequency. Audit documentation for all centres in an international clinical trial is therefore not always easy to obtain and heterogeneous in modality and frequency of execution: the heterogeneous regulatory landscape makes what should be a straightforward requirement - provide proof of proper beam output audit - into a source of delay and an extra cost for institutions, and ultimately a barrier to access to trials for patients.

While an internal audit could be a good starting point, a centralised national/external audit program should be established. The general impression at the European level was that implementation of clinical audits were not in general pushed forward by the countries in the process of transposition of the Council Directive into national legislation and that there might have been an undesirable overlap with inspections. Moreover, the role of the Medical Physicist into clinical audits needed to be ascertained.

This prompted EFOMP to initiate a survey to identify what kind of audit, either clinical or dosimetry is mandated by law in different European countries and which role is played by the Medical Physicist. This work in particular aimed to give an overview of regulations and practices for periodical QA and QC on RT equipment in EFOMP member states, just as EU countries have taken different steps to implement the Council Directive 2013/59/EURATOM [1].

In this work, one of the main objectives of EFOMP was to harmonise and promote the best practice of Medical Physics within Europe. Under this paradigm, EFOMP supports the requirements of the Council Directive 2013/59/EURATOM of 5 December 2013 [1] in which medical physicists have a high level of competence and clear definition of duties and responsibilities. EFOMP also endorses the Council Directive'es statement that medical physics experts are recognised by the competent authorities. According to it: "Member States shall ensure that: the practitioner, the medical physics expert and those entitled to carry out practical aspects of medical radiological procedures are involved, as specified by Member States, in the optimisation process" (art 57(1)(b)), and "Member States shall ensure that: in medical radiological practices, a medical physics expert is appropriately involved, the level of involvement being commensurate with the radiological risk posed by the practice and in particular: in radiotherapeutic practices other than standardised therapeutic nuclear medicine practices, a medical physics expert shall be closely involved" (art 58(d)).

In parallel with the daily clinical environment, the value of international harmonization and dissemination of best practices in clinical trials cannot be understated, in particular when it comes to QA in a technology-driven field such as Radiation Oncology. The adoption of common, harmonized dosimetry procedures internationally would not only improve safety, but also facilitate the setup and conduct of RT clinical trials for academic sponsors, increasing access opportunities for patients. This work will also help to assess the level of heterogeneity in dosimetry audit requirements across EFOMP and EU member states, a necessary first step in the process of simplifying trial participation and patient enrolment in clinical trials.

\section{Methods}

The questionnaire was jointly designed to describe the overall general audit standards in RT for dosimetry QA and periodical dosimetric checks across EU countries. Therefore, ten general questions about audit requirements for dosimetric QA and qualifications needed to be enrolled in clinical trials were included in the questionnaire.

The questionnaire was sent to 33 NMOs in November of 2019 (by the time the survey was spread, 33 NMO were part of the EFOMP). Results were collected during the period December 2019 - March 2020. The questionnaire was sent to NMO Presidents and EFOMP's delegates, and bi-weekly reminders were sent during this period. Additionally, in March 2020 final individual requests were also sent to collect pending answers. All the answers were collected through the Wufoo platform, an open-source web-based application for surveys. The actual survey received by NMOs representatives can be seen in Supplementary Material A.

\section{Results}

Members of 19 out of 33 EFOMṔs NMOs responded to the survey (58\% response rate, $14 \mathrm{EU}$ members (54\%) vs. 5 non-EU members (46\%)). The questionnaire targeted the medical physics community to evaluate the regulatory status of dosimetry audits carried out in RT departments.

The analysed results are presented below without disclosing the name of the national member organisation. Data concerning each country that participated in the survey was kept confidential and not disclosed with the results of this survey.

Requirements of periodic dosimetry audits for radiotherapy and type of auditor

The first section of the questionnaire enquired about the requirements of periodic dosimetry audits for RT departments across Europe, the entity (auditor) performing the audit according to national laws, as well as the source of the auditor (internal/external).

In $58 \%$ of the countries participating in the questionnaire (11/19 NMOs, $9 \mathrm{EU}$ members and 2 non-EU members) national regulations required regular dosimetry audits to be carried out in RT departments (Fig. 1). In these countries, the audit was required by law to be carried out either by an internal auditor e.g. the hospital Medical Physics Department (3 NMOs), or by an external auditor (7 NMOs) which was either a specific auditing institution solely authorised to perform such audits in the country (such as the National Cancer Center or the National Atomic Energy Agency) or was selected from a list of multiple entities authorised for executing such quality checks. These entities varied across countries, but they were mostly external Medical Physics Experts authorised by the competent authority through the local professional body.

Among those $42 \%$ of the countries stating that regular dosimetry audits were not regulated on a national level (8/19 NMOs, 5 EU members and 3 non-EU members): one member state reported that the audit is conducted by an external auditor, one -by an internal auditor, while the rest reported that the type of the audit was either not specified by law or it could be carried out by anyone with a minimum required certification.

\section{Parameters and procedures to be followed for the validity of the audit}

The survey further aimed to identify the existence of any precisely established parameters and procedures (established by national legislation or by the national Medical Physics Associations/Colleges or equivalent bodies) to be followed for the validity of the audit. In case of the existence of a separate third entity evaluating or validating the results, the NMOs were requested to specify its identity.

Of the 11 NMOs reporting that national regulations require regular dosimetry audits for RT departments, 5 stated that there were wellestablished procedures in place to be followed for the audit to be valid. These procedures were set by either national legislation (2 NMOs), the national Medical Physics association (1 NMO), or by accredited external auditors ( 2 NMOs). In most situations, the same entity executing quality checks also produces the analysis and proceeds with validation/invalidation. 


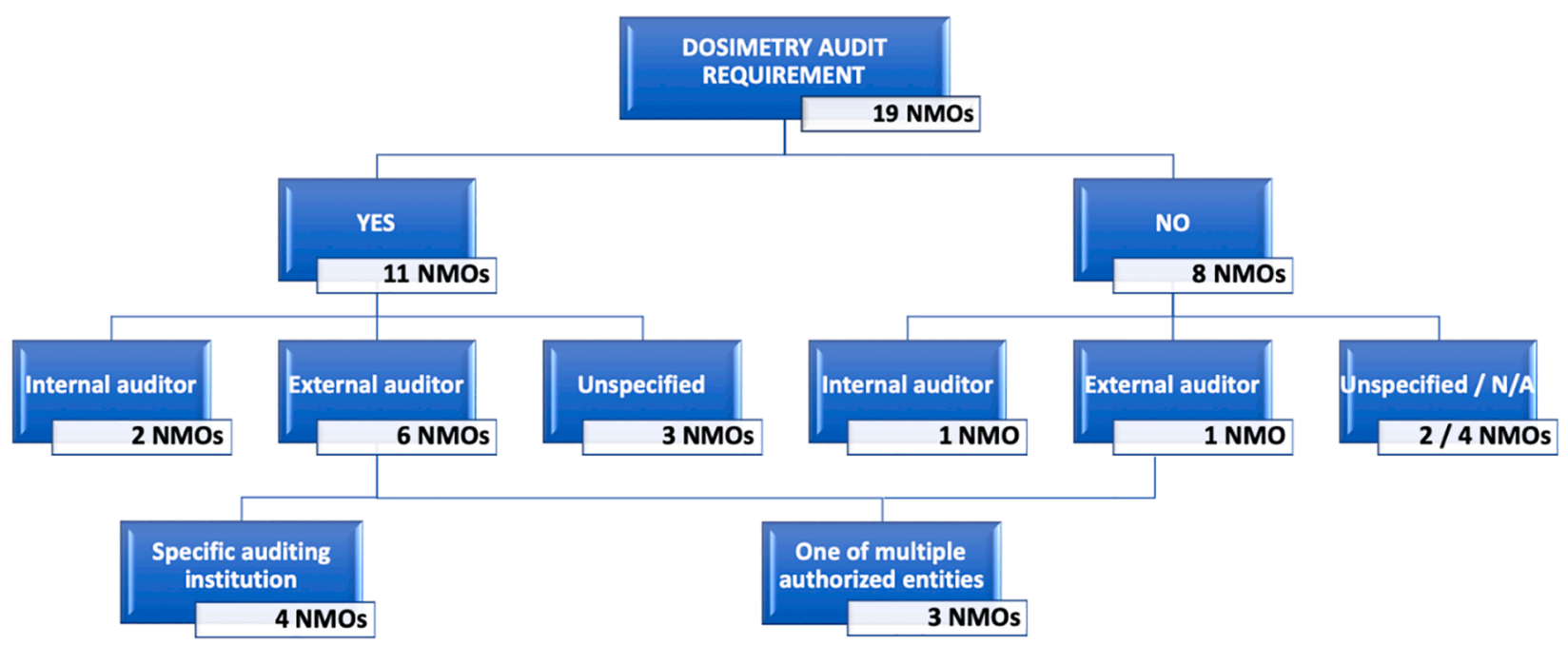

Fig. 1. Dosimetry audit requirements according to the NMOs' responses.

Dosimetry standards for credited departments to treat patients

The last part of the survey focused on basic reference dosimetry requirements, frequency, and specific instrumentation to be credentialed for treating patients. Overall, while not always regulated by national legislation, all responding countries had implemented dosimetry procedures following either national or international guidelines [4-6].

Regulations about required QA frequencies and instrumentation varied widely within and across countries. In few countries a QA Program must be presented to the Health Authority for approval, which should also specify the instrumentation and frequency of the procedures. There were common answers found among NMOs related to reference dosimetry procedures in RT departments and periodic dosimetric checks:

- Prior to the first clinical use of the RT facility a quality audit must be performed (independent intercomparison of source calibration).
- Acceptance testing and constancy testing must be carried out in accordance with international standards.

- Reference dosimetric procedures were usually defined according to international dosimetric protocols (IAEA, AAPM) [4-6].

- Instrumentation suggested by international/locally adapted protocols must be used and calibrated for absolute dose measurements every two years. Dosimetric equipment should be metrologically traceable to the national standard maintained by the National Dosimetry laboratory.

More specifically, for advance or technique-specific procedures the answers reflected that in $63 \%$ of the participating countries (12/19 NMOs, $7 \mathrm{EU}$ members vs. 5 non-EU members) there were no mechanisms regulating minimum dosimetric QA requirements or needed instrumentation to be used. In $37 \%$ of the participating countries $(7 / 19$ NMOs, all EU members) there were implemented regulations for independent/secondary dose verification: two countries reported mandatory independent dose calculation, two countries reported mandatory in vivo

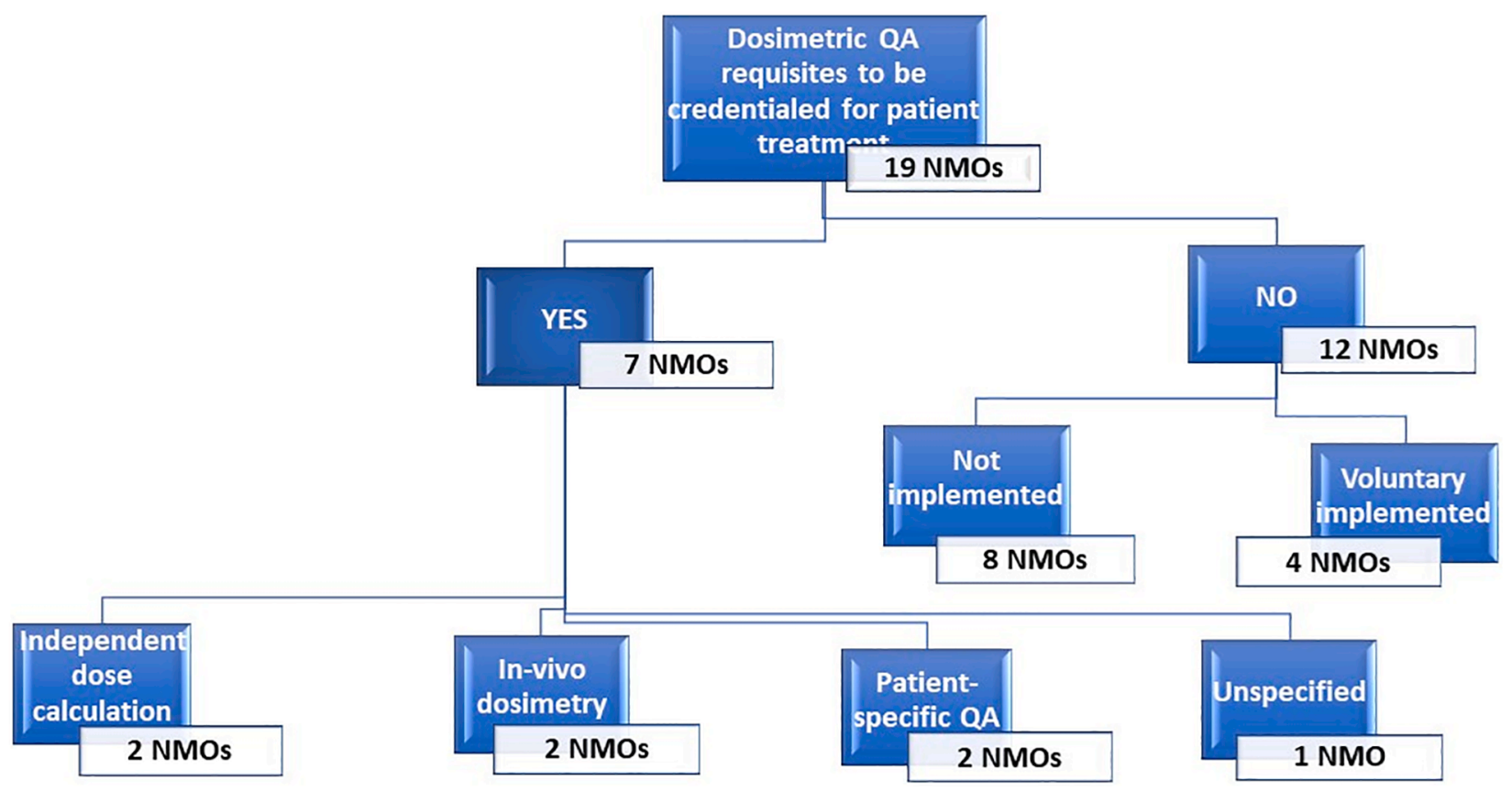

Fig. 2. NMO's answers for dosimetric QA requisites to be credentialed to treat patients. 
dosimetry, two countries reported mandatory pre-treatment volumetric dose measurements, while one country reported patient-specific QA accordingly with international standards. In four countries (4 NMOs) similar procedures were already implemented although it was not regulated by the competent authorities and rely on the discretion of the medical physics experts (Fig. 2).

For $58 \%$ of the participating countries (11/19 NMOs, 7 EU members vs. 4 non-EU members) there were no mechanisms to ensure or/and review regular updates with technology evolution. For $42 \%$ of the participating countries (8/19 NMOs, 7 EU members vs. 1 non-EU member) there were implemented procedures to review updates and advances in the field of RT.

\section{Specific requirements to participate in clinical trials}

Overall, there was no specific regulation for participation in clinical trials. In $37 \%$ of the participating countries (7/19 NMOs, 3 EU members vs. 4 non-EU members) it was not mandatory to use a CEE marked TPS, while in the $58 \%$ of the participating countries (11/19 NMOs, $10 \mathrm{EU}$ members vs. 1 non-EU member) it was always mandatory to use a CEE marked TPS, regardless any involvement in clinical trials. One NMO (EU member) did not complete this question.

Secondly, dose-engine validation for all the countries participating in the questionnaire (19 NMOs, $14 \mathrm{EU}$ members vs. 5 non-EU members) relied on international recommendations [4-6], with no specific requirements for patient planning related to participations in clinical trials. Only one NMO reported that some clinical trials might entailed minimum TPS/dose-engine validation requirements for enrolment. Furthermore, $42 \%$ of the participating countries (8 NMOs) also reported that TPS/dose-engine validation was linked to TPS commissioning during acceptance, typically following vendors verification tests and/or international guidelines [4-7].

Finally, $84 \%$ of the participating countries (16 NMOs, 11 EU members vs. 5 non-EU members) reported that no special legal requirements by the competent authority were requested to carry out clinical research in RT. In contrast $16 \%$ of the participating countries (2 NMOs, EU members) reported that special legal requirements were needed to carry out clinical research.

\section{Discussion}

Dosimetry audits are one of the key measurements implemented to ensure patient safety in RT. Council Directive 2013/59/EURATOM emphasized the role of dosimetric audits and gave directions for their implementations accordingly to national procedures. Therefore, differences in the implementation of dosimetric audits across European countries became an important point of interest for EFOMP, who sought for addressing dosimetric audit requirements for participation in clinical trials, and harmonization of clinical practice across European countries. This survey has served to picture differences in requirements for dosimetric audits in Europe.

Overall, this survey evidenced a significant heterogeneity in the national policies about dosimetric audits in RT departments, even though with only a partial coverage of the sample under scrutiny. Although transposition into national legislation of the Council Directive 2013/59/EURATOM came applicable in 2018, by the end of 2019 dosimetric audits were only implemented in $58 \%$ of the countries participating in the questionnaire. There were also differences in the agent required to carry out the audits: internal auditor, external auditor or unspecified (Fig. 1).

Similar results can be found in other domains of medical radiological procedures. Recent surveys led by the European Society of Radiology (ESR) amongst European radiology departments and national radiology societies have confirmed that Basic Safety Standard Directive (BSSD) compliance, integration of processes of clinical audit/re-audit and the development of external audit networks remained inconsistent across member states.

Notwithstanding the almost completed transposition into national legislation of the 2013/59/EURATOM Council Directive, prompted through article 58(e), the establishment of clinical audits for all the medical radiological procedures was scarce. The present survey evidenced that only 9 out of the $18 \mathrm{EU}$ members confirmed that national legislation required regular dosimetry audits to be carried out in RT departments. Indeed, a work carried out in a tender in the BSSD's pretransposition period, awarded by the European Commission to a consortium made up by EFOMP, ESR and the European Federation of Radiographer Societies (EFRS), demonstrated that Member States continued to experience difficulties in implementing clinical audit in radiological practices.

This situation prompted the European Commission's Directorate General for Energy to fund the QuADRANT project, led by the European Society of Radiology together with the European Association of Nuclear Medicine (EANM) and the European Society for Radiotherapy and Oncology (ESTRO) as consortium partners, which started in January 2020. The QuADRANT aims to review the status of implementation of clinical audit in EU member states, to improve its broader use and develop guidance and recommendations, beside the ones already existing [8-11]. The QuADRANT is divided into work packages which include: 1) The organisation of a workshop on clinical audit of radiology, radiotherapy and nuclear medicine practice, which was run as a series of webinars in December 2020; 2) A survey into the implementation of clinical audit in the member states, which is currently ongoing, 3) The organisation of a workshop to present the Member's states results scheduled in 2021, and 4) The preparation of recommendations, which should be released within the terms for the completion of the project (30 months). Although EFOMP is not directly involved in the management of the projects, it was asked to nominate three Medical Physics Experts in the Executive Board and is therefore actively committed to the success of this initiative.

Other international initiatives in the field which are worth mentioning are the audit program of radiotherapy practices established by the International Atomic Energy Agency (QUATRO), which from 2005 to 2019 organized 96 missions (38 in Europe) and the Dose audits for Radiotherapy centres organized since 1969 by the same International Atomic Energy Agency, and which involved so far 2300 radiotherapy centres in 133 states.

EFOMP believes that only a multi-stakeholder approach could be successful in promoting the culture of audit in the clinical environment. Therefore, we are actively seeking the collaboration with our "sister" organisations involved in the clinical specialties and we are participating in all the initiatives that could bring the practice of the clinical audit where it should be from where it is today.

From the point of view of a clinical trial organization, the heterogeneities observed in this work can be put in historical perspective: the EORTC mailed dosimetry program results indicated in the past that the majority of dose deviations $>3 \%$ presented themselves in institutions who did not participate in frequent external dosimetry audits $(>5 \mathrm{y})$ [12]. More importantly, it was concluded that small deviations in beam output could lead to tumour control probability losses up to $8 \%$ and an increase in mild to moderate morbidity up to $22 \%$ [13]. Such variations would represent an unacceptable and, more importantly, preventable source of variation in RT clinical trials with the potential to unpower the whole trial [14]. Large, multi-centric international trials would benefit greatly from a more harmonized approach to dosimetry audits across EU and EFOMP members.

On the contrary, a significant element of homogeneity in the survey is the role of the Medical Physics Experts in the participation of both internal and external audits. This was somewhat expected due to the role that the 2013/59/EURATOM Council Directive assign to medical physicists as the sole responsible of the dosimetric aspects of the RT treatment. Additionally, a working group has been created within EFOMP to further evaluate the role of Medical Physicists, to cover all 
current and emerging areas of the Medical Physicist's role in clinical trials and to complement any guidance from National Member Organisations [15].

EFOMP was actively participating in both processes on one side through the participation of Medical Physics Experts in the executive board of QuADRANT project and on the other side by updating the core curriculum in Radiotherapy for the Medical Physics Experts in a joint collaboration with ESTRO and by providing Certification for the National Registration Schemes for Medical Physics Expert recognition at the National level [15].

\section{Conclusion}

The transposition and further implementation of the 2013/59/ EURATOM Council Directive through national legislation is scarce, leading to heterogeneities in the national policies about dosimetric audits in RT.

\section{Acknowledgements}

We would also like to thank all the NMOs representatives who carefully replied to this survey, for sharing and providing useful information for the medical physics community.

\section{References}

[1] European Parliament. Council Directive 2013/59/Euratom of 5 December 2013 laying down basic safety standards for protection against the dangers arising from exposure to ionising radiation, and repealing Directives 89/618/Euratom, 90/641/ Euratom, 96/29/Euratom, 97/43/Euratom a. Off J Eur Commun L13 2014:1-73.

[2] E. Parliament C. Directive 97, 43, Euratom of 30, on health protection of individuals against the dangers of ionizing radiation in relation to medical exposure, and repealing Directive 84/466/Euratom Off J Eur Commun L13 1997 June 19972227.

[3] Melidis C, Bosch WR, Izewska J, Fidarova E, Zubizarreta E, Ulin K, et al. Global harmonization of quality assurance naming conventions in radiation therapy clinical trials. Int J Radiat Oncol Biol Phys 2014;90:1242-9. https://doi.org/ 10.1016/j.ijrobp.2014.08.348.

[4] IAEA. Quality Assurance in Radiotherapy. IAEA TECDOC No. 989. 1998. https:// www.iaea.org/publications/5644/quality-assurance-in-radiotherapy (accessed November 27, 2020).

[5] IAEA. Absorbed Dose Determination in External Beam Radiotherapy. Technical Reports Series No. 398. 2000. https://www.iaea.org/publications/5954/absorbeddose-determination-in-external-beam-radiotherapy (accessed November 27, 2020).

[6] Almond PR, Biggs PJ, Coursey BM, Hanson WF, Huq MS, Nath R, et al. AAPM's TG51 protocol for clinical reference dosimetry of high-energy photon and electron beams. Med Phys 1999;26:1847-70. https://doi.org/10.1118/1.598691.

[7] Fraass B, Doppke K, Hunt M, Kutcher G, Starkschall G, Stern R, et al. AAPM's TG-53 quality assurance for clinical radiotherapy treatment planning. Med Phys 1998;25: 1773-829. https://doi.org/10.1118/1.598373.

[8] Directorate-General for Energy and Transport. No 159-European Commission guidelines on clinical audit for medical radiological practices (diagnostic radiology, nuclear medicine, and radiotherapy) Radiation Protection, 2009. https://doi.org/10.2768/20266.

[9] IAEA. Quality Management Audits in Nuclear Medicine Practices 2015. https:// www.iaea.org/publications/10714/quality-management-audits-in-nuclearmedicine-practices (accessed December 21, 2020).

[10] IAEA. Comprehensive Clinical Audits of Diagnostic Radiology Practices: A Tool for Quality Improvement 2010. https://www.iaea.org/publications/8187/ comprehensive-clinical-audits-of-diagnostic-radiology-practices-a-tool-for-qualityimprovement (accessed December 21, 2020).

[11] IAEA. Comprehensive Audits of Radiotherapy Practices: A Tool for Quality Improvement 2007. https://www.iaea.org/publications/7680/comprehensiveaudits-of-radiotherapy-practices-a-tool-for-quality-improvement (accessed December 21, 2020).

[12] Dutreix A, van der Schueren E, Derreulnaux S, Chavaudra J. Preliminary results of a quality assurance network for radiotherapy centres in europe. Radiother Oncol 1993;29:97-101. https://doi.org/10.1016/0167-8140(93)90232-W.

[13] Bentzen SM, Bernier J, Davis JB, Horiot JC, Garavaglia G, Chavaudra J, et al. Clinical impact of dosimetry quality assurance programmes assessed by radiobiological modelling of data from the thermoluminescent dosimetry study of the european organization for research and treatment of cancer. Eur J Cancer 2000; 36:615-20. https://doi.org/10.1016/S0959-8049(99)00336-6.

[14] Weber DC, Tomsej M, Melidis C, Hurkmans CW. QA makes a clinical trial stronger: evidence-based medicine in radiation therapy. Radiother Oncol 2012;105:4-8. https://doi.org/10.1016/j.radonc.2012.08.008.

[15] EFOMP: European Federation of Organizations for Medical Physics. https://efomp. org/ (accessed January 2, 2021). 